\title{
ESTUDIOS SOBRE LA ESCHERICHIA COLI
}

\section{I. - INVESTIGACION DE LAS CEPAS D433, BETA Y 026 B6 EN LAS DIARREAS AGUDAS NO ESPECIFICAS DEL LACTANTE. Comunicación Preliminar.}

\author{
Drs. MANUEL RODRIGUEZ, JULIO MENEGHELLO y Sr. R. ADASME \\ Cátcdra Extraordinaria de Pediatria. Prof. Jalio Meneghello. Hospitan M. Arriaran. \\ Universidad de Chile. Instituto de Microbiología e lomunología. Prof. H. Vaccaro.
}

La etiologia de los cuadros diarreicos agudos del niño, constituye un problema en permanente estudio. Entre los agentes infecciosos, capaces de alterar la normalidad del tubo digestivo, podríamos decir que, junto a las Salmonellas y Shigellas, reconocidamente patógenas para el intestino, hay una gran variedad de bacterias y virus, cuyo papel en la etiología de estos cuadros clínicos recién comienza a dilucidarse.

Es así como dentro del grupo de bacterios considerados habitualmente saprófitos del intestino, se han ido demostrando variedades de Proteus, Klebsiellas y últimamente Escherichias, cuya asociación con los procesos diarreicos agudos y con epidemias de diarreas, sugieren un papel activo en el trastorno de la fisiologia y anatomía normal del tubo digestivo.

En 1944 Baevan $^{1} \mathrm{y}$ un año más tarde Bray ${ }^{2}$, pudieron demostrar la presencia de algunas cepas de Escherichia Coli en cuadros de enteritis agudas del niño, llamando particularmente la atención que, la presencia de estas cepas daban, tanto a los cultivos de ella como a las deposiciones mismas, un olor característico a semen. Esta cepa fué identificada más tarde con el nombre de Bacilo Coli Napolitanum.

Con posterioridad a estas primeras comunicaciones sobre el problema, muchas otras han venido a confirmar este hallazgo bacteriológico en las diarreas agudas no especificas, destacándose entre ellas las hechas por Taylor y col. ", Giles y col. ${ }^{455}$, Shanks y col. "i, Stevenson ', Laurell y col." y Braun y col. 5 .

En un estudio realizado por Kauffmann y Dupont en $1950^{10}$ de una serie de 33 cepas, aisladas principalmente en Europa, se pudo establecer dos tipos fundamentales de antígenos somáticos y de superficie los cuales fueron designados 055-0111 y B4-B5, respectivamente. Respecto a las cepas móviles, en sus cilios se demostró la existencia de tres antígenos diferentes designados: $\mathrm{H} 2-\mathrm{H} 6$ y $\mathrm{H} 12$.

De la combinación de estos antígenos resultan dos cepas prototipo: a) O111 B4 H2, con un subtipo 0111 B4 H12 y b) 055 B5 H6. Posteriormente un tercer prototipo de estructura antigénica somática fué descrita correspondiendo a la fórmula 026 B6.

Dada las distintas denominaciones que se encuentran en la literatura, especialmente para el prototipo a), resumimos en el cuadro $N$ ? las sinonimias.

Creemos que no es necesario analizar la frecuencia con que los diferentes investigadores han encontrado este germen asociado con las diarreas inespecíficas del niño, por lo que sólo nos referiremos a la comunicación que en 1948 hicieron Bray $\mathrm{y}$ Beavan ${ }^{11} \mathrm{y}$ cuyos resultados en este sentido exponemos en el cuadro $\mathrm{N}^{Q} 2$.

Respecto a los hechos que apoyan el valor que como agentes etiológicos de las diarreas agudas no específicas del niño tienen estas cepas, diferenciándose así del B. Coli común, Netter y col. ${ }^{12}$ han establecido lo siguiente:

1. En el curso de una epidemia este tipa serológico de Coli está presente en la mayoria de los pacientes.

2. El tipo serológico de Coli, es el germen predominante en las deposiciones de los enfermos.

$$
\text { CLALT) Na } 1
$$

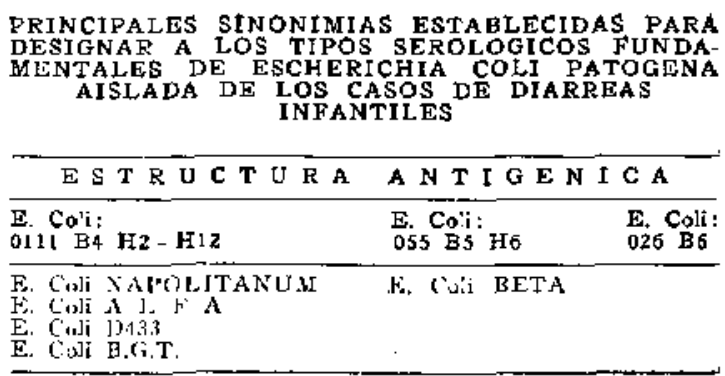


C.LADRO NO 2

FRECUENCIA DE AISLAMIENTO DE LAS CEPAS D433. BETA Y 026 B5 EN PACIENTES DE GASTRO. ENTERITIS Y EN CONTROLES EN LOS ANOS 1943 Y 1945 EN EUROPA (*)

\begin{tabular}{|c|c|c|c|c|c|}
\hline \multirow[b]{2}{*}{ Ā̄o } & \multicolumn{3}{|c|}{ Gastronteritis } & \multicolumn{2}{|c|}{ Controles } \\
\hline & $\begin{array}{l}\text { Ne de } \\
\text { casos }\end{array}$ & $\begin{array}{l}\% \text { de Bac. } \\
\text { D4 } 33 \\
\text { aistado }\end{array}$ & $\begin{array}{l}\% \text { de mot- } \\
\text { talidad }\end{array}$ & $\begin{array}{l}\text { No de } \\
\text { Casas }\end{array}$ & $\begin{array}{c}\% \text { de Bac. } \\
\text { D433 } \\
\text { ais!ado }\end{array}$ \\
\hline $\begin{array}{l}194,3 \\
19+5\end{array}$ & $\begin{array}{l}511 \\
411\end{array}$ & $\begin{array}{l}95,1) \\
87,3\end{array}$ & $\begin{array}{l}311,0 \\
23.0\end{array}$ & $\begin{array}{r}100 \\
80\end{array}$ & $\begin{array}{l}4,0 \\
4.0\end{array}$ \\
\hline$(*)$ & $\begin{array}{l}\text { Citida te } \\
\text { and Bact. }\end{array}$ & $\begin{array}{l}B+3 y=J \\
60: 395-401\end{array}$ & $\begin{array}{l}\text { I Beitriu, } \\
9+3 .\end{array}$ & E. D, & \\
\hline
\end{tabular}

3. Estos gérmenes se encuentran raramente en nin̆os o adultos sanos.

4. Administrado a voluntarios humanos, adultos y niños, da origen a diarrea grave.

5. Después de administrar a aduitos dosis elevadas de $B$. Coli patógeno, se ha demostrado en estos sujetos la presencia de aglutininas y hemoaglutininas, en cambio esto no sucede al ser administrado el $\mathrm{B}$. Coli común.

Finalmente, los hechos epidemiológicos en relación al mecanismo de transmisión del germen dentro de una sala de hospital, han contribuído fuertemente a demostrar el papel de este tipo serológico en los cuadros clínicos que comentamos.

En nuestro medio, es posible observer $r$ en las lactantes epidemias de diarrea cuya etiología no es posible establecer aún en las mejores condiciones de estudio bacteriológico. Del mismo modo muchos casos de diarreas agudas del lactante quedan sin una etiología precisa, aun cuando se estudien satisfactoriamente desde el
CUAJRO XQ

TRATAMIENTO RECIBIDO POR S6 LACTANTES CON DIARREA AGUDA EN LOS QUE SE INVESTIGARON LAS CEPAS D433, BETA Y O26 B6 DE LA ESCHERICHIA COLI

\begin{tabular}{|c|c|c|c|c|c|c|}
\hline \multirow[b]{2}{*}{ Diagnóstico } & \multicolumn{2}{|c|}{ Estreptomicina } & \multicolumn{2}{|l|}{$\begin{array}{l}\text { Cloroam- } \\
\text { fenicol }\end{array}$} & \multirow[b]{2}{*}{$\begin{array}{l}\text { Sin } \\
\text { trat. * }\end{array}$} & \multirow[b]{2}{*}{ Tota? } \\
\hline & $\begin{array}{l}100 \mathrm{~m} \\
\text { c. } 8 \mathrm{~b} \text {. }\end{array}$ & $\begin{array}{l}200 \mathrm{~m} \text {. } \\
\text { c. } 8 \mathrm{~h} .\end{array}$ & $\begin{array}{l}250-500 \\
\text { mgr. d. }\end{array}$ & $\begin{array}{l}\text { Trata- } \\
\text { corrb. }\end{array}$ & & \\
\hline $\begin{array}{l}\text { Enterocolitis } \\
\text { Dispepsias }\end{array}$ & 9 & 3 & $\stackrel{3}{a}$ & $\begin{array}{l}4 \\
8\end{array}$ & $\frac{2}{3}$ & $\begin{array}{l}13 \\
38\end{array}$ \\
\hline TOTALES & 20 & $*$ & 11 & 12 & 5 & 56 \\
\hline
\end{tabular}

punto de vista bacteriológico $\mathrm{y}$ se descarten otros factores. Estos hechos nos indujeron a realizar un plan de investigaciones Inmuno-Bacteriológicas, tanto en niños como en adultos, acerca de la participación de estas cepas en las diarreas de causa no específica cuyos resultados parciales damos a conocer en esta ocasión, en una comunicación preliminar, considerando el valor que en el estudio de estos enfermos tiene esta comprobación.

\section{MATERIAL Y MÉTODOS}

Sólo nos referiremos a grandes rasgos a las características y metódica empleada en este estudio.

\section{Material clínico.}

Nuestro material comprende 76 casos de los cuales 49 son varones y 27 mujeres, cuyas edades están comprendidas en un rango de 1 día a 2 años con un promedio de 6,0 meses y una desviación standard de 5,4 .

CUADRO NiQ 4

FRECUENCIA DE AISLAMIENTO DE LAS CEPAS D433, BETA Y 026 B6 EN LACTANTES SANOS Y PACIENTES CON SINDROME DIARREICO AGUDO

\begin{tabular}{|c|c|c|c|c|c|c|c|c|}
\hline \multirow[b]{2}{*}{ Casos } & \multicolumn{3}{|c|}{ Escherichia Coli } & \multicolumn{2}{|c|}{$\begin{array}{l}\text { Otros agentes } \\
\text { concomitantes }\end{array}$} & \multirow{2}{*}{$\begin{array}{l}\text { Más de una } \\
\text { cepa de } E \text {, Coli }\end{array}$} & \multirow{2}{*}{ Negativos } & \multirow{2}{*}{ Total } \\
\hline & D433 & Beta & $025 \mathrm{BS}$ & $\begin{array}{l}\text { Shig. " } \\
\text { Salci. }\end{array}$ & Parásitos & & & \\
\hline \multirow{2}{*}{ Enteracoltis } & 3 & 3 & 2 & 0 & $1 *$ & 5 & 4 & 18 \\
\hline & $(16.350)$ & $(16,8 \%)$ & $(11,1 \%)$ & $(0.1) \%)$ & $(5.4 \%)$ & $(27,7 \%)$ & $(23,2 \%)$ & $(100,0 \%)$ \\
\hline \multirow{2}{*}{ Jispensias } & 3 & 5 & 4 & ( & 0 & 4 & 22 & 38 \\
\hline & $(7,8 \%$ & $(13,4 \%)$ & $(10,5 \%)$ & $\{[1,010\}$ & $(19,6 \%)$ & $(10,5 \%)$ & $(27,8 \%)$ & $(1110,0 \%)$ \\
\hline \multirow{2}{*}{ Sanos } & 0 & 0 & 0 & 0 & 0 & ) & $2 n$ & 20 \\
\hline & $(0,0 \%)$ & $(0,0 \%)$ & $(0,0 \%)$ & $(0,0 \%)$ & $(11,0 \%)$ & $(0,0 \%)$ & $(100,0 \%)$ & $(100,0 \%)$ \\
\hline \multirow{2}{*}{ TOTAI.ES } & 6 & 8 & 8 & 0 & 1 & 9 & 4t: & 76 \\
\hline & $\left(H, 1 r_{n}\right)$ & $(10,5 \%)$ & (10,5 $)$ & $(0,0 \%)$ & $(1,3 \%)$ & $(11.0 \%)$ & $(f, n, 5 \%)$ & $(100,0 \approx)$ \\
\hline
\end{tabular}

* Slrigellas - - Salrnonelias.

* Asociación de E. Coli Beta con I anuljas rutestimalis y Entamueba Histolítica. 
CTAADRO Ni

FRECUENCIA DEL AISLAMIENTO DE LAS CEPAS D433, BETA Y O26 BG EN LACTANTES SANOS Y EN PACIENTES CON SINDROME DIARREICO AGUDO

\begin{tabular}{|c|c|c|c|}
\hline Canos & $\begin{array}{c}\text { Ny } \\
\text { pasitivos }\end{array}$ & Porcentaje & $\begin{array}{l}\text { Ny de casos } \\
\text { estudiados }\end{array}$ \\
\hline $\begin{array}{l}\text { Wuterucolití } \\
\text { Wistreprias } \\
\text { Sallati }\end{array}$ & $\begin{array}{r}14 \\
10 \\
10\end{array}$ & $\begin{array}{l}77,7 \div \\
42,0 \\
11,0\end{array}$ & $\begin{array}{l}13 \\
38 \\
20\end{array}$ \\
\hline TOTAIES & 30 & 53,5 & 26 \\
\hline
\end{tabular}

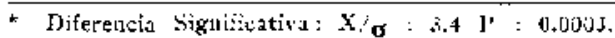

De este grupo de niños, 18 fueron catalogados como Enterocolitis por presentar diarrea con mucus, pus y sangre al examen microscópico de las deposiciones y 38 fueron catalogadas como Dispepsias, ya que presentaban diarrea simple sin carácter inflamatorio, proceso que por lo general acompañaba a un cuadro febril de tipo infeccioso, con un grado más o menos variable de deshidratación, que en algunos casos asumió el carácter típico de la Toxicosis. 20 niños sanos fueron escogidos como control.

Los casos de diarreas agudas fueron obtenidos del Servicio de Lactantes de la Sección "A" de Medicina del Hospital Manuel Arriarán. Lactantes de la Policlínica del Hospital y niños sanos pertenecientes a grupos privados, constituyen los grupos de control.

En todos estos nin̄os se practicó un coprocultivo tomando la muestra en pequeños frascos ad-hoc, las que fueron en lo posible sometidas de inmediato a cultivo, como también una muestra de secreción faríngea.

\section{Procedimiento Bacteriológico.}

Ambas muestras fueron sembradas en Agar lactosado tornasolado, medio de Mac Conkey's y Levine. A las 24 horas de incubación, las colonias de $\mathbf{E}$. Coli, en número de 4-6 por placa, fueron sometidas a la aglutinación en lámina y tubos mediante sueros inmunes $0 B$ obtenidos en conejos mediante la inoculación de las cepas tipo $\mathrm{D} 433$, Beta y 026 B6 las cuales nos fueron gentilmente enviadas por la Dra. Joan Taylor del Salmonella Reference Laboratory de Londres.

Las colonias que aglutinaron fueron traspasadas a agar simple para su conservación $y$ estudios posteriores desde diferentes aspectos.

Las muestras fueron tomadas lo más precozmente posible en relación al mo-
〔บUIJRO X? 6

NUMERO DE CASOS EN LOS QUE SE AISLARON SIMULTANEAMENTE DOS CEPAS DIFERENTES DE ESCHERICHIA COLI

\begin{tabular}{|c|c|c|c|}
\hline Cabras & $\begin{array}{c}\text { D433 } \\
026 \quad \text { B6 }\end{array}$ & $\begin{array}{c}\text { Beta } \\
026 \text { B6 }\end{array}$ & $\begin{array}{l}\text { D433 } \\
\text { Beta }\end{array}$ \\
\hline $\begin{array}{l}\text { Euterenonlitis } \\
\text { Dispepsias } \\
\text { Sincsios }\end{array}$ & $\begin{array}{l}1 \\
0 \\
0\end{array}$ & $\begin{array}{l}1 \\
2 \\
0\end{array}$ & $\begin{array}{l}3 \\
1 \\
0\end{array}$ \\
\hline TOTALES & 1 & 3 & 4 \\
\hline
\end{tabular}

mento en que se hospitalizó el niño, con el fin de evitar el tratamiento antibiótico previo, sin embargo, no siempre fué esto posible ya que un buen grupo de ellos alcanzaron a recibir una o dos dosis de Estreptomicina o Cloroamfenicol previa a la toma de la muestra. En el cuadro $\mathrm{N}^{\circ} 3$ podemos apreciar el uso de antibióticos en nuestros pacientes.

Los 5 casos no tratados previamente corresponden a enfermos a los cuales se les alcanzó a tomar la muestra en el momento de ingreso al Servicio sin que hubieran recibido ningún antibiótico previo.

\section{Hallazgos bacteriológicos.}

En los cuadros No 4 y 5 podemos apreciar los resultados bacteriológicos de nuestro material.

Los porcentajes de aislamiento de las cəpas de E. Coli patógena, como puede apreciarse en el cuadro No 4 son estrechamente semejantes sin observarse una tendencia significativa a predominar un tipo sobre el otro. Sólo en el grupo de ninos con dispepsia se pudo apreciar un ligero predominio de las cepas Beta y 026 B6 sobre la D433.

El análisis global de los casos positivos en los dos grupos de enfermos, como se puede apreciar en el cuadro No 5 nos demuestra un franco y significativo predominio de los casos positivos a E. Coli patógena en el grupo de las enterocolitis en relación a los casos positivos para el mismo grupo de gérmenes de los casos de Dispepsias. El análisis estadístico de esta diferencia reveló un $\mathrm{x} / \boldsymbol{\sigma}$ de 3,4 con un $\mathrm{P}$ de 0,0003 , lo cual es significativo. Es de la mayor importancia destacar, además, que en ninguno de nuestros controles de niños sanos encontramos cepas de Escherichia Coli patógena, como puede apreciarse en ambos cuadros y lo cual, sin duda, es un hecho de gran interés cuando se comparan estos resultados con los hallazgos en el enfermo. 
CUADRO N*

NUMERO DE CASOS EN LOS QUE SE AISLARON SIMULTANEAMENTE LAS CEPAS D433, BETA Y 026 B 6 EN EL EXUDADO FARINGEO Y EN LA DEPOSICION

\begin{tabular}{|c|c|c|c|c|c|}
\hline Casog & D433 & Beta & $026 \mathrm{BG}$ & $\begin{array}{l}\text { D433 } \\
\text { Beta }\end{array}$ & $\begin{array}{c}\text { Beta } \\
026 \mathrm{~B} 6\end{array}$ \\
\hline $\begin{array}{l}\text { Equterocolitis } \\
\text { Jjisplepsias } \\
\text { Sinlos }\end{array}$ & $\begin{array}{l}0 \\
0 \\
0\end{array}$ & $\begin{array}{l}1 \\
1 \\
0\end{array}$ & $\begin{array}{l}0 \\
1 \\
0\end{array}$ & $\begin{array}{l}1 \\
0 \\
0\end{array}$ & $\begin{array}{l}\text { t } \\
0 \\
0\end{array}$ \\
\hline TOTAIES & 0 & 2 & 1 & 1 & 1 \\
\hline
\end{tabular}

Sólo en un caso, perteneciente al grupo de los niños con Enterocolitis, encontramos simultáneamente con una cepa de $\mathrm{E}$. Coli Beta, Entamoebas Histolíticas y Lamblias Intestinalis. Este fué un enfermo de 10 meses de edad con una evolución tórpida y prolongada y con profundos signos físicos de Distrofia. A excepción de este caso no observamos ninguna otra asociación de bacterias patógenas o de parásitos, aún en repetidos cultivos de control.

Podemos finalmente apreciar que, en el grupo de las Enterocolitis, en un 27,7\% de ellas se aislaron simultáneamente más de una cepa de E. Coli patógena de las deposiciones y en el grupo de las Dispepsias se observó esta asociación en el $10,5 \%$. En el cuadro No 6 destacamos el tipo de asociación encontrada en estos casos.

Respecto a la asociación del Coli común con e platógeno, en una misma deposición, con el patógeno, en una misma deposición, tivos obtuvimos escasas colonias del Coli común al lado del patógeno, apreciándose siempre el Coli patógeno en cultivo puro o en un predominio muy marcado sobre el Coli común o saprófito.

Dijimos anteriormente que en nuestro plan contemplamos la investigación simultánea de estas cepas en las deposiciones y en la secreción faringea. Los resultados se pueden apreciar en el cuadro No 7.

En nuestros casos no encontramos nunca algunas de estas tres cepas en la secreción faríngea exclusivamente, sino que siempre en forma simultánea en la secreción faríngea $\mathrm{y}$ en la deposición, coincidiendo, además, el tipo de cepa aislada en ambas muestras, aún en los casos en los que hubo asociación de cepas. Finalmente nuestros controles sanos tampoco presentaron Coli patógenos en sus secreciones faringeas.

Quisimos además, evaluar la relación que pudiera existir entre el estado de gravedad del cuadro clínico y el hallazgo de la E. Coli patógena en las deposiciones.
CUADRO NO $B$

FRECUENCTA DEL AISLAMIENTO DE LAS CEPAS D433, BETA Y 026 B6 EN RELACION CON LA GRAVEDAD DEL SINDROME DIARREICO

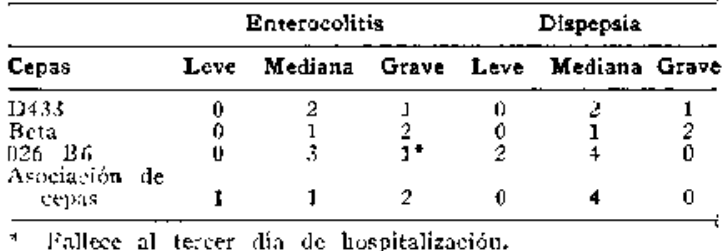

Los resultados podemos apreciarlos en el cuadro No 8.

No se señaló una franca relación o tandencia en los casos estudiados, probablemente llame la atención que sólo una minoría correspondieron a casos leves, los demás pudieron catalogarse entre mediana gravedad y graves. Un solo caso de Enterocolitis, de 7 meses de edad, ingresó en extremo grado de gravedad y en él se aisló una Escherichia Coli 026 B6, falleciendo al cuarto día de ingreso y encontrándose en la autopsia un proceso úlcerohemorrágico que cubría la última porción del intestino delgado y gran parte del grueso. Todos los otros casos evolucionaron, desde el punto de vista del trastorno intestinal, en forma satísfactoria, permaneciendo hospitalizados plazos variables en relación con el grado de Distrofia que presentaban.

\section{DISCUSIÓN}

Los diferentes trabajos publicados en relación a la presencia de la $\mathrm{E}$. Coli patógena en casos de diarreas y vómitos de carácter epidémico en lactantes, han demostrado desde distintos puntos de vista el papel que estas cepas desempeñan como agente etiológico de este síndrome.

En esta comunicación preliminar, sobre el problema en nuestro medio hemos podido establecer un porcentaje de aislamiento del germen muy semejante al obtenido por autores extranjeros, especialmente europeos. Por otra parte hemos podido apreciar un significativo predominio de estos gérmenes en los casos de Enterocolitis en relación con los casos de Dispepsias $\mathrm{y}$, lo que es más importante, que aun cuando nuestro grupo de niños sanos es reducido, no encontramos en ellos este tipo serológico de Coli.

Es muy llamativo el hallazgo de un Coli Beta en la deposición y secreción farín- 
gea de un prematuro que ingresó a las 24 horas de nacer con una deposición francamente purulenta $y$ en la que no se encontró, fuera del Coli Beta otro agente etiológico. Esto, sin duda, apoyaría la opiniión de Rogers ${ }^{13}$ en el sentido de la transmisión directa del portador al caso, ya sea por intermedio de instrumentos o aparatos de uso en los pabellones donde se colocan estos niños o directamente por las manipulaciones del personal que los atiende. Finalmente en nuestro caso tan particular existe la posibilidad de la transmisión de la madre, como una portadora, hacia el niño, en el momento del parto.

La comprobación anátomo-patológica de lesiones úlcero-hemortágicas de la mucosa del intestino delgado y grueso en los fallecidos $\mathrm{y}$ en los que en vida se pudo aislar una E. Coli patógena, nos parece un buen argumento, junto a los ya citados, para justificar el papel que se atribuye a estas cepas, en los trastornos diarreicos agudos del lactante.

\section{RESUMEN}

1) Se relata una comunicación preliminar acerca de la investigación de las cepas del bacilo Coli D433, Beta y 026 B6 en las diarreas agudas no específicas del lactante, como parte de una más amplia investigación clínico-bacteriológica y epidemiológica del problema.

2) Se estudian 76 casos, correspondientes a: 18 Enterocolitis típicas, 38 Dispepsias y 20 lactantes sanos como control, pudiéndose comprobar:

a) Una positividad global deI $\mathbf{7 7 , 7} \%$ para las Enterocolitis y del $42,0 \%$ para las Dispepsias, diferencia que es estadísticamente significativa. En cambio, en los controles sanos esta investigación fué negativa.

b) No se apreció una tendencia a predominar significativamente alguna de as tres cepas estudiadas en los dos grupos de enfermos. Tampoco fué posible establecer una relación entre la gravedad de la afección y el tipo de germen aislado.

c) En 5 casos se aisló simultáneamente la misma cepa en la secreción faríngea y en la deposición.
3) Se discute el valor de estos hallazgos en la etiología de las diarreas no específicas del lactante.

\section{SUMMARY}

1. A preliminary comunication on the investigation of the different strains of the Coli bacillus, D433, Beta and 026 B6 in the acute non-specified diarrheas of the infant, is related, as part of a greater clinical-bacteriological and epidemiological investigation of the problem.

2. 76 cases are studied corresponding to: 18 typical Enterocolitis, 38 Dispepsias and 20 healthy infants as controls. The following results were obtained:

a) A global positivity of $77.7 \%$ for the Enterocolitis and $42.0 \%$ for the Dispepsias, which difference is important statistically. On the other hand, in the healthy controls, this investigation was negative.

b) No tendency to a significant predcminance of any of the three strains studied in both groups of patients was seen. Neither was it possible to establish a relationship between the seriousness of the disease and the type of germ isolated.

c) In 5 cases there was simultaneous isolation of the same strain in the pharynx secretion and in the stools.

3 . The value of these findings in the ethiology of the non-specific diarrheas of infants is discussed.

\section{BIBLIOGRAFÍA}

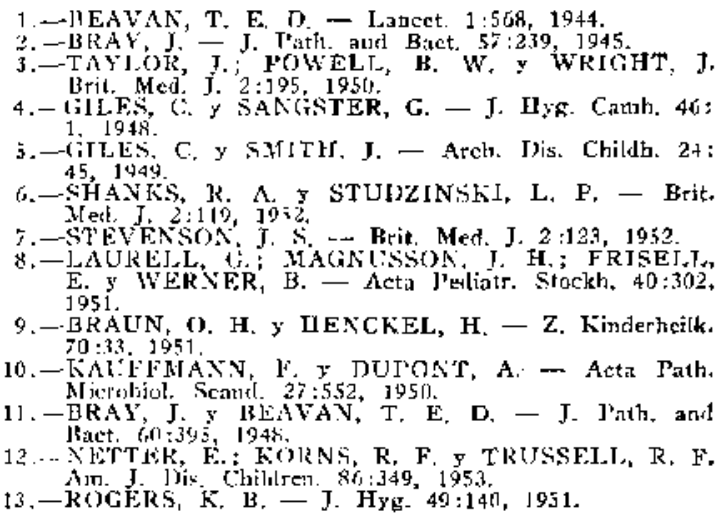

